Supplementary Information for:

\title{
Metal-Organic Framework Photoconductivity via Time-Resolved Terahertz Spectroscopy
}

Brian Pattengale, ${ }^{\dagger, \S}$ Jens Neu, ${ }^{\ddagger}$, Sarah Ostresh, ${ }^{\dagger}$ Gongfang $\mathrm{Hu},{ }^{\dagger}$ Jacob A. Spies, ${ }^{\dagger}$ Ryotaro Okabe, ${ }^{\dagger, \perp}$ Gary W. Brudvig, ${ }^{\dagger}$ and Charles A. Schmuttenmaer ${ }^{\dagger}$

tDepartment of Chemistry and Yale Energy Sciences Institute, Yale University, New Haven, Connecticut 06520-8107

‡Department of Molecular Biophysics and Biochemistry and Yale Microbial Sciences Institute, Yale University, New Haven, Connecticut 06520-8107

\section{Current Address:}

¿School of Life Science and Technology, Tokyo Institute of Technology, Nagatsuta 4259, Midori-ku, Yokohama, Kanagawa 226-8503, Japan

Table of Contents:

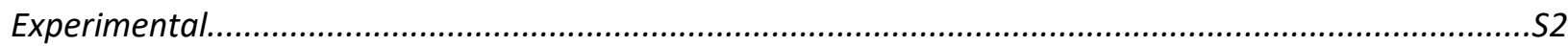

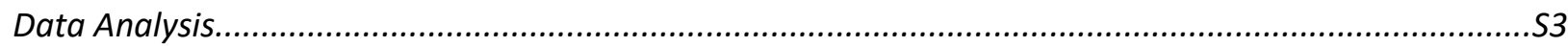

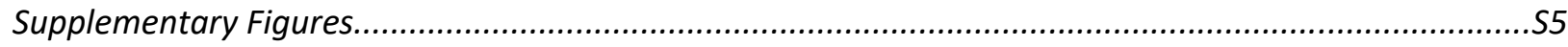

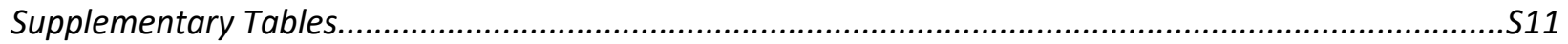

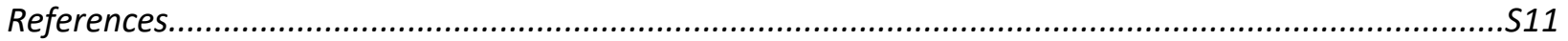




\section{Experimental}

\section{Synthesis and Characterization of $\mathrm{H}_{4}$ TTFTB}

$\mathrm{H}_{4}$ TTFTB (tetrathiafulvalene tetrabenzoate) was synthesized following the literature procedures without modification. ${ }^{1,2}$

\section{Synthesis and Characterization of $\mathrm{Zn}_{2}$ TTFTB}

$\mathrm{Zn}_{2}$ TTFTB was synthesized according to the published procedure. ${ }^{2} \mathrm{~A}$ ligand solution was prepared in a 50 $\mathrm{mL}$ round bottom flask by dissolving $100 \mathrm{mg}$ of $\mathrm{H}_{4}$ TTFTB in $12 \mathrm{~mL}$ of dimethylformamide, followed by the slow addition of $4 \mathrm{~mL}$ of ethanol. In a separate vessel, $159.5 \mathrm{mg}$ of $\mathrm{Zn}\left(\mathrm{NO}_{3}\right)_{2} \cdot 6 \mathrm{H}_{2} \mathrm{O}$ was dissolved in $8 \mathrm{~mL}$ of ethanol followed by the addition of $8 \mathrm{~mL}$ of dimethylformamide. A Teflon stir bar was added to the flask containing the ligand solution and was used to stir gently. The metal solution was then added to the ligand solution slowly, with no precipitation formed. The flask was evacuated and $\mathrm{N}_{2}$-filled three times on a Schlenk line, and was then heated to $75^{\circ} \mathrm{C}$ for 48 hours. The dark precipitate was collected by vacuum filtration and washed three times with dimethylformamide followed by ethanol. pXRD patterns were obtained using a Rigaku SmartLab X-ray diffractometer. Reference pXRD data was calculated using Mercury (CCDC). UV-Visible measurements were performed using a Shimadzu UV-2600 spectrometer with integrating sphere attachment.

\section{Tape Cell Preparation}

A "tape cell" is formed by using a standard hole punch (diameter $7 \mathrm{~mm}$ ), to make a hole in a standard paper business card (thickness $0.35 \pm 0.01 \mathrm{~mm}$ ). This allows delicate powder samples to be studied without having to press a pellet or sinter the sample. The acrylic tape used in this study was Intertape Polymer Group model number 291 (thickness $2.5 \mathrm{mil}=63.5 \mu \mathrm{m}$ ). A small rectangular piece of business card with a hole punch near the end was used to prepare a tape cell (see main text Figure $1 \mathrm{~b}$, inset). The paper piece was covered on one side with a piece of acrylic tape. The cell was then laid upside down on a flat surface with the empty hole facing upwards. Powder MOF sample was added to the hole, followed by using the flat edge of a metal spatula against the paper of the cell to fill the hole to the top and lightly scrape away excess. This process was repeated 3 times, and excess powder was then removed. The cell was sealed with a second piece of acrylic tape. The excess tape was trimmed from the cell, and isopropanol was used to wipe clean the outside of the cell prior to measurement. After measurement, the thickness of the tape-sample-tape layer was determined using a micrometer.

\section{THz Spectroscopy}

Complex-valued refractive index data were collected using a THz time-domain spectrometer (THz-TDS) as described elsewhere. ${ }^{3-5}$ Briefly, the spectrometer utilizes the output of a $300 \mathrm{~mW}$ Titanium Sapphire oscillator, with a pulse-repetition rate of $90 \mathrm{MHz}$, operating at $800 \mathrm{~nm}$ center frequency. The ultra-short laser pulses (sub $30 \mathrm{fs}$ ) are split in a ratio of 95:5. The stronger beam is focused on an interdigitated antenna, which is biased with $10 \mathrm{~V}$. This bias is modulated with $4.2 \mathrm{kHz}$ and the modulation frequency is used as master-clock of a lock-in amplifier. The second portion of the output beam is routed by a mechanical delay line and focused on a dipole antenna with $10 \mu \mathrm{m}$ gap. Both antennas are fabricated on LT-GaAs. Samples were mounted in a translation sample holder for micro-positioning. THz transmission 
as a function of sample position was collected for the purpose of checking sample homogeneity. Air reference and tape references were collected for determination of sample refractive indices. Using these three measurements the complex refractive index of the sample was determined as described in our recent publication. ${ }^{3}$ These calculations solved the complex transmission equations numerically. No model assumptions have been used, in particular the calculation is carried out without invoking a Kramers-Kronig relation by directly retrieving the complex transmission function obtained from the transmitted $\mathrm{THz}$ wave and reference.

Photoconductivity data were collected using an amplifier-based time-resolved THz spectrometer described elsewhere. ${ }^{6-8}$ Briefly, the $800 \mathrm{~nm}$ fundamental of a Ti:Sapphire chirped-pulse regenerative amplifier (Spectra-Physics Spitfire Ace, $35 \mathrm{fs} \mathrm{fwhm,} 1 \mathrm{kHz}$ repetition rate, $3.5 \mathrm{~mJ}$ pulse energy) was split into three beams for $\mathrm{THz}$ generation, $\mathrm{THz}$ detection, and optical excitation, respectively. $\mathrm{THz}$ radiation was generated in a two-color air plasma ${ }^{9,10}$ and subsequently detected via electro-optical sampling in ZnTe (110). ${ }^{11}$ The optical excitation beam (pump) was frequency doubled to $400 \mathrm{~nm}$ in a $\beta$-barium borate crystal. For optical-pump THz-probe (OPTP) experiments, the time delay between the optical excitation pulse and $\mathrm{THz}$ pulse is controlled using a mechanical delay line. For time-resolved $\mathrm{THz}$ spectroscopy (TRTS) experiments where the frequency-dependent complex conductivity is determined, another mechanical delay line controls the time delay between the $\mathrm{THz}$ generation and detection pulses. Three tape cell samples were measured. In general, data is collected by chopping the excitation pulses and using a lock-in amplifier referenced to the chopping frequency to collect a difference spectrum in time domain. Then, the $\mathrm{THz}$ generation line is chopped to collect a reference spectrum for the nonphotoexcited sample. The photoexcited time domain trace can then be calculated by adding the difference spectrum to the non-photoexcited spectrum.

\section{Data Analysis}

THz-TDS data processing:

In order to describe the photoinduced changes accurately, the static, unaltered refractive index must be determined first. This was done as described in our recent publication. ${ }^{3}$ Note: all of these quantities are complex-valued. In short, THz-TDS provides the frequency-resolved amplitude and phase of an electromagnetic wave. Therefore, the sample measurement can be referenced to an air measurement to determine the phase change and the amplitude change simultaneously. This complex valued transmission function $T_{\text {exp }}$ is then compared with the known geometry of the sample (illustrated in Figure S2) which is described by $T_{\text {calc }}$ as shown below:

$T_{\text {calc }}=\tau_{0 t} P_{t} \tau_{t M} P_{M} \tau_{M t} P_{t} \tau_{t 0} P_{0}^{-1}$

The subscripts describe air (0), the tape (t) and the MOF (M), where $\tau_{i j}=\frac{2 n_{i}}{n_{i}+n_{j}}$ is the Fresnel amplitude transmission coefficient for an electromagnetic wave entering medium $j$ coming from medium $i . P_{i}$ describes the propagation in the medium $i$. This operator accounts for the phase delay and absorption in the medium in addition to the internal reflection in the cell:

$P_{i}=e^{-\mathrm{i} k_{0} n_{i} d_{i}} \sum_{\mathrm{m}=0}^{\infty}\left(\rho_{j i} \rho_{i k} e^{-2 \mathrm{i} k_{0} n_{i} d_{i}}\right)^{m}=\frac{e^{-i k_{0} n_{i} d_{i}}}{1+\rho_{j i} \rho_{i k} e^{-2 \mathrm{i} k_{0} n_{i} d_{i}}}$ 
Where $\rho_{i j}=\frac{n_{i}-n_{j}}{n_{i}+n_{j}}$ is the Fresnel reflection coefficient, $k_{0}=\frac{\omega}{c}$ is the wavevector in vacuum, and $d_{i}$ is

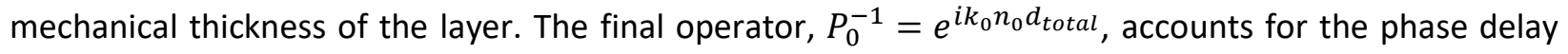
the wave experiences in the absence of a sample. An opposite sign is used in the exponential to account for the fact that vacuum is replaced by sample material in the experiment.

This equation was numerically solved using the optimization tool box in MATLAB. Analysis programs are available for download on our group website at thz.yale.edu

\section{TRTS data analysis:}

A similar approach is used to solve for the photoconductive refractive index, as described in detail previously and illustrated in Figure S2. ${ }^{12}$

In short, the transmission function through the sample without photoexcitation is defined analogously to $T_{\text {calc }}$ in equation 1:

$T_{\text {off }}=\tau_{0 t} P_{t} \tau_{t M} P_{M} \tau_{M t} P_{t} \tau_{t 0} P_{0}^{-1}$

The photoexcited case is described with:

$T_{\text {on }}=\tau_{0 t} P_{t} \tau_{t M} P^{\prime}{ }_{M} \tau_{M M}^{*} P_{M}^{*} \tau_{M t}^{*} P_{t} \tau_{t 0} P_{0}^{-1}$

Where the ${ }^{*}$ denotes photoexcited material. The thickness of the MOF is split in $d_{M}=d^{\prime}+\delta$ where $\delta$ is the photoexcited layer, defined by the penetration length and $d^{\prime}$ is the non-photoexcited layer.

The experimentally measured transmission with and without photoexcitation $E_{o n} / E_{o f f}$ is then compared with the equations:

$\frac{T_{o n}}{T_{o f f}}=\frac{P_{M}^{\prime} \tau_{M M}^{*} P_{M}^{*} \tau_{M t}^{*}}{P_{M} \tau_{M t}}==\frac{E_{o n}}{E_{o f f}}$

It is important to note that the thickness of the tape cell is not part of the final equation, hence small variations caused by fabrication inaccuracy and similar effects will not interfere with the refractive index determination.

In a final step, the refractive index for the on and off cases are compared to derive the photoconductivity:

$n_{o n}-n_{o f f}=\Delta n=\sqrt{\frac{\mathrm{i} \Delta \sigma}{\omega \epsilon_{0}}}$

Where $\epsilon_{0}$ denotes the vacuum permittivity and $\sigma$ denotes the photoconductivity.

The photoconductivity data was smoothed in Origin Pro using the Lowess algorithm with a $0.15 \mathrm{THz}$ span. The smoothed data was then linearly interpolated to reduce the number of data points for clarity.

The Drude-Smith model was used to fit the complex photoconductivity data: ${ }^{13,14}$

$\sigma_{D S}(\omega)=\frac{N e^{2} \tau_{\text {scatt }} / m^{*}}{1-i \omega \tau_{\text {scatt }}}\left(1+\frac{c_{1}}{1-i \omega \tau_{\text {scatt }}}\right)$ 
Where $N$ is the carrier density, $e$ is the electron charge, $m^{*}$ is the effective mass of the charge, and $\omega$ is the angular frequency of the $\mathrm{THz}$ probe. The term $c_{1}$ accounts for the effects of grain boundaries.

Data analysis programs are available free of charge under GNU-license from our homepage: http://thz.yale.edu

OPTP data analysis:

OPTP data were fit with a Gaussian convoluted triexponential function in Origin Pro.

$\Delta T H z=\left[\sum_{i=1}^{n} A_{i} \exp \left(-\frac{t_{\text {pump }}-t_{0}}{\tau_{i}}\right)\right] \otimes G_{r}(f w h m)$

It was found that three decay constants were necessary to adequately describe the decay. The Gaussian FWHM IRF (full-width at half-maximum instrument response function) was set to $0.6 \mathrm{ps}$ to account for the pulse width of the $\mathrm{THz}$ field and the pump-beam. During the fitting process, the longest time constant $\left(\tau_{3}\right)$ was fixed to a value much longer than the data time window to account for remaining signal (10 ns).

\section{Supplementary Figures}
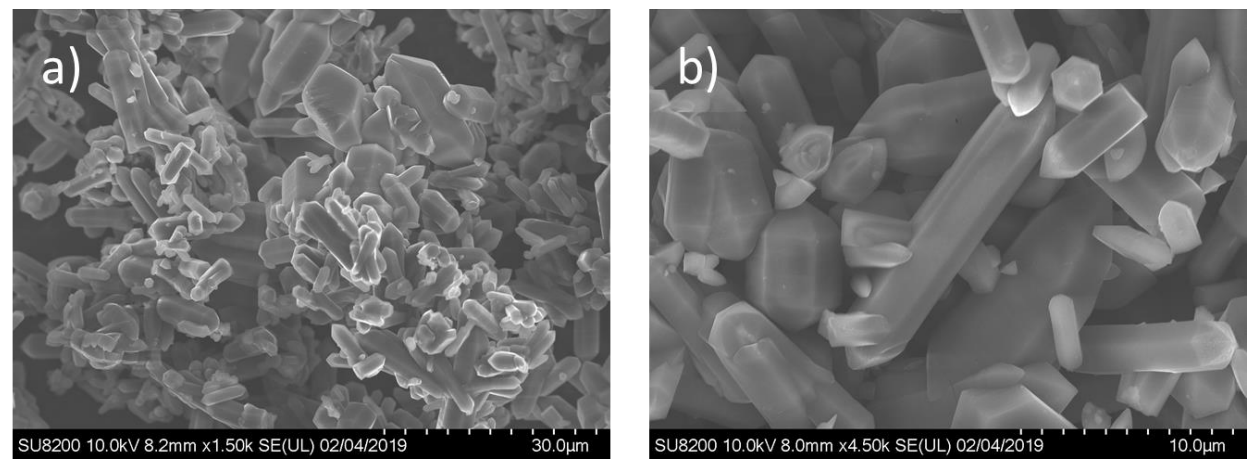

Figure S1. SEM images of a polycrystalline $Z n_{2}$ TTFTB sample illustrating the morphology of MOF crystallites and their size. 


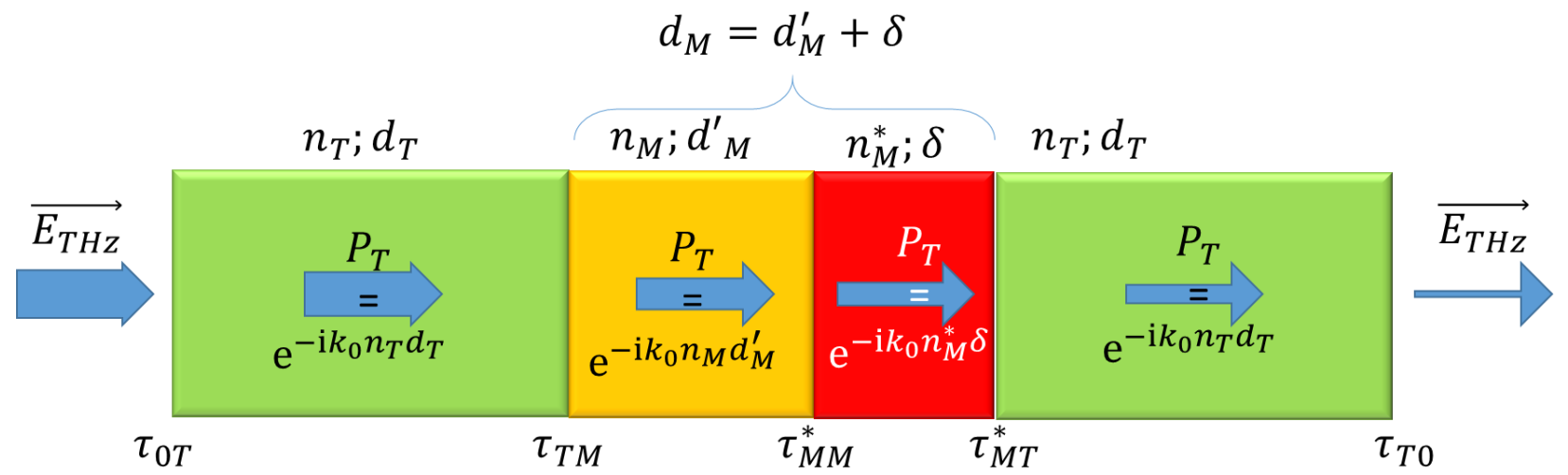

Figure S2. Scheme depicting the photoexcited transmission function used in refractive index calculations. For TDS (the non-photoexcited sample), the photoexcited layer (red) is replaced with nonexcited material (orange). The transmission coefficient between these two identical layers is then 1 , and is treated as a single layer.
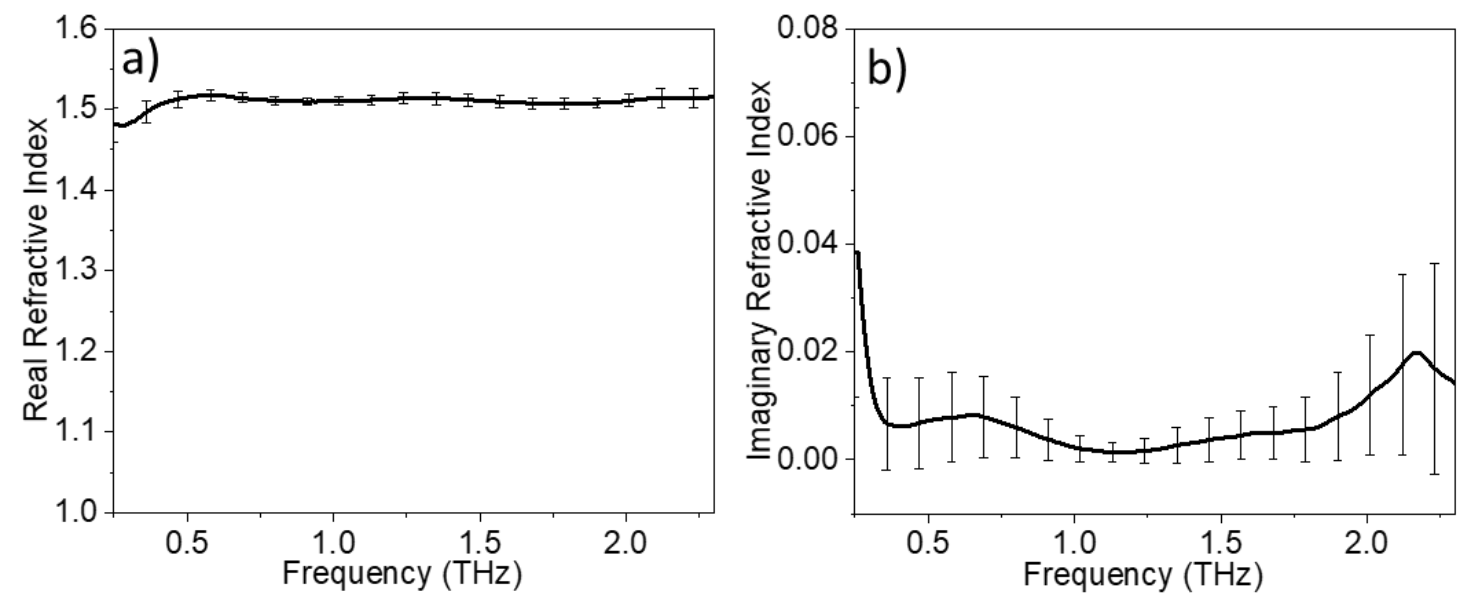

Figure S3. Real (a) and imaginary (b) parts of the refractive index for the tape cell reference, without sample illustrating a flat refractive index across our measurement bandwidth. 

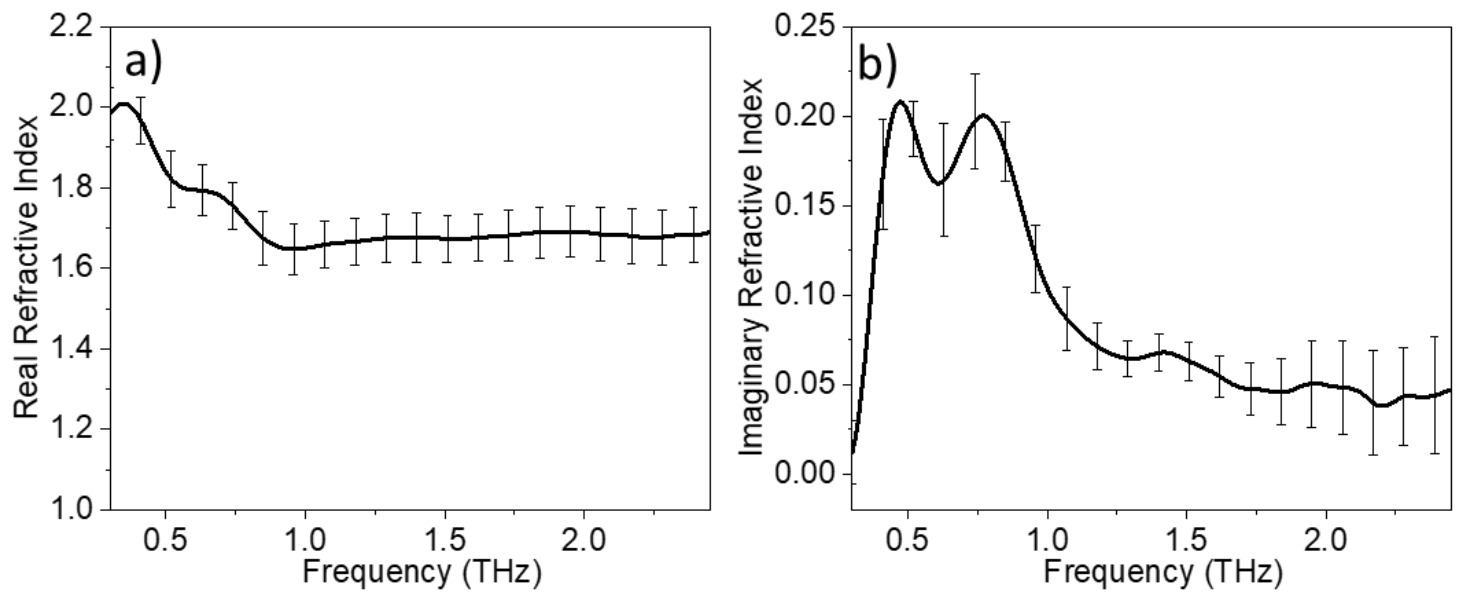

Figure S4. Real (a) and imaginary (b) parts of the refractive index for the $\mathrm{Zn}_{2}$ TTFTB sample pressed with 11 kbar pressure, showing a splitting of the resonance compared to the unpressed material, shown in the main part of the manuscript.
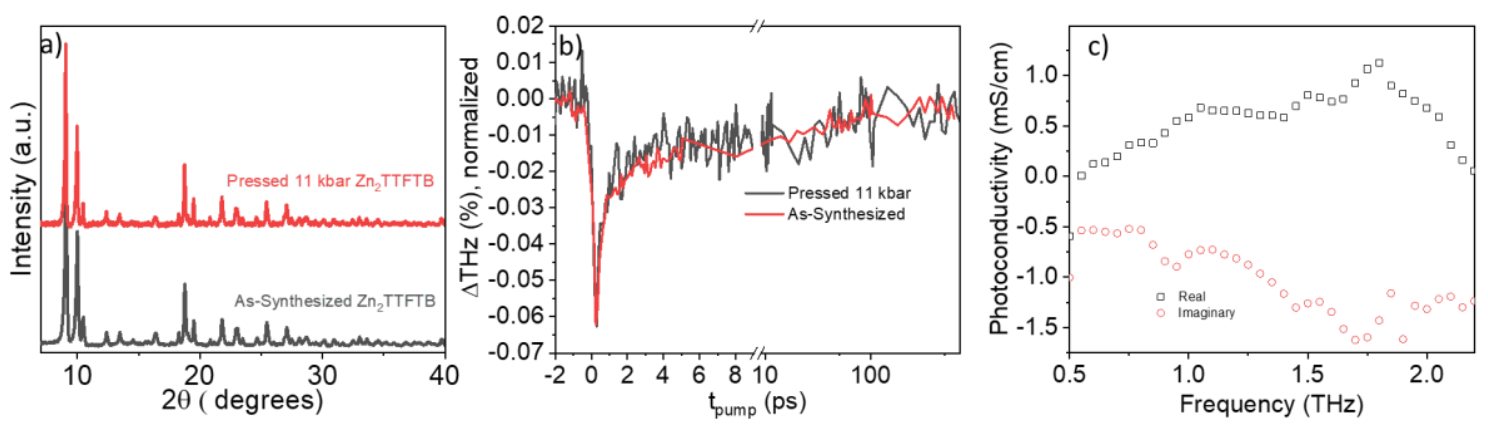

Figure S5. (a) pXRD of $\mathrm{Zn}_{2}$ TTFTB extracted from tape cells after applied pressure of $11 \mathrm{kbar}$. We note no observable changes in the bulk structure. The normalized OPTP dynamics (b) show no changes due to applied pressure. The frequency-resolved TRTS data (c) show a slight increase in magnitude for the pressed sample, particularly in the ranve of 1.5 to $2.0 \mathrm{THz}$, which we ascribe to improved grain-to-grain contact in the pressed sample. 

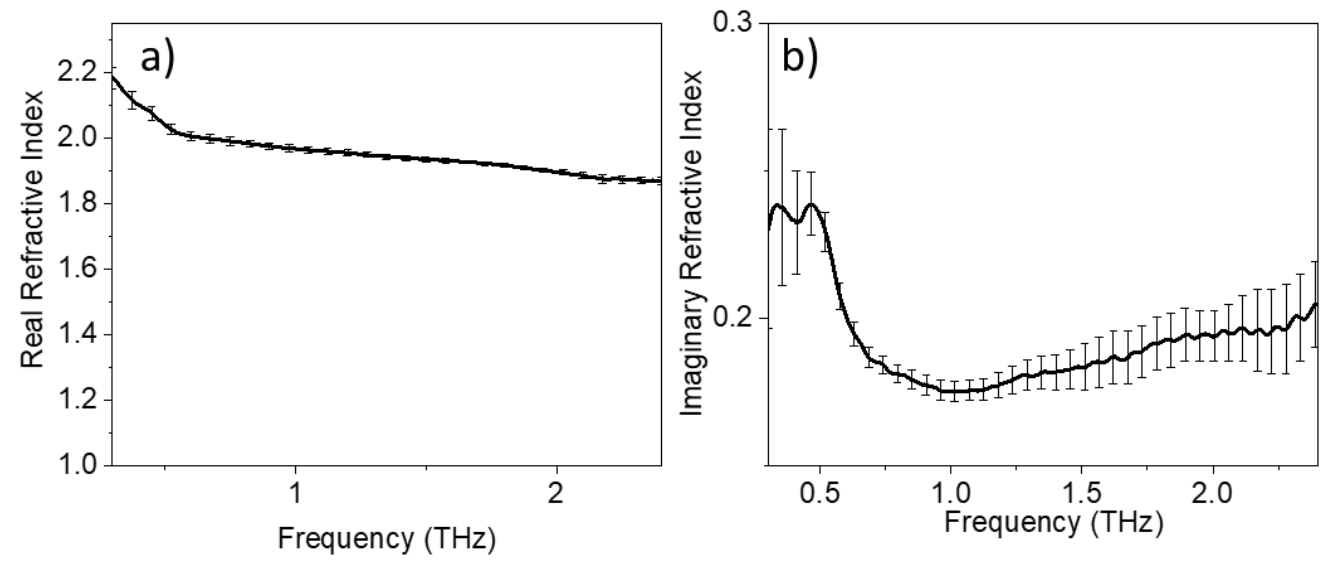

Figure S6. Real (a) and imaginary (b) parts of the refractive index for a pressed pellet $Z \mathrm{n}_{2}$ TTFTB sample in PTFE (11 kbar). The resonant absorption feature observed in (b) shows a shift compared to the tape cell sample of approximately $0.1 \mathrm{THz}$ to lower frequency. However, such a pressed sample is not usable for photoconductivity experiments because the MOF is embedded in a PTFE matrix that does not transmit the $400 \mathrm{~nm}$ excitation pulse.
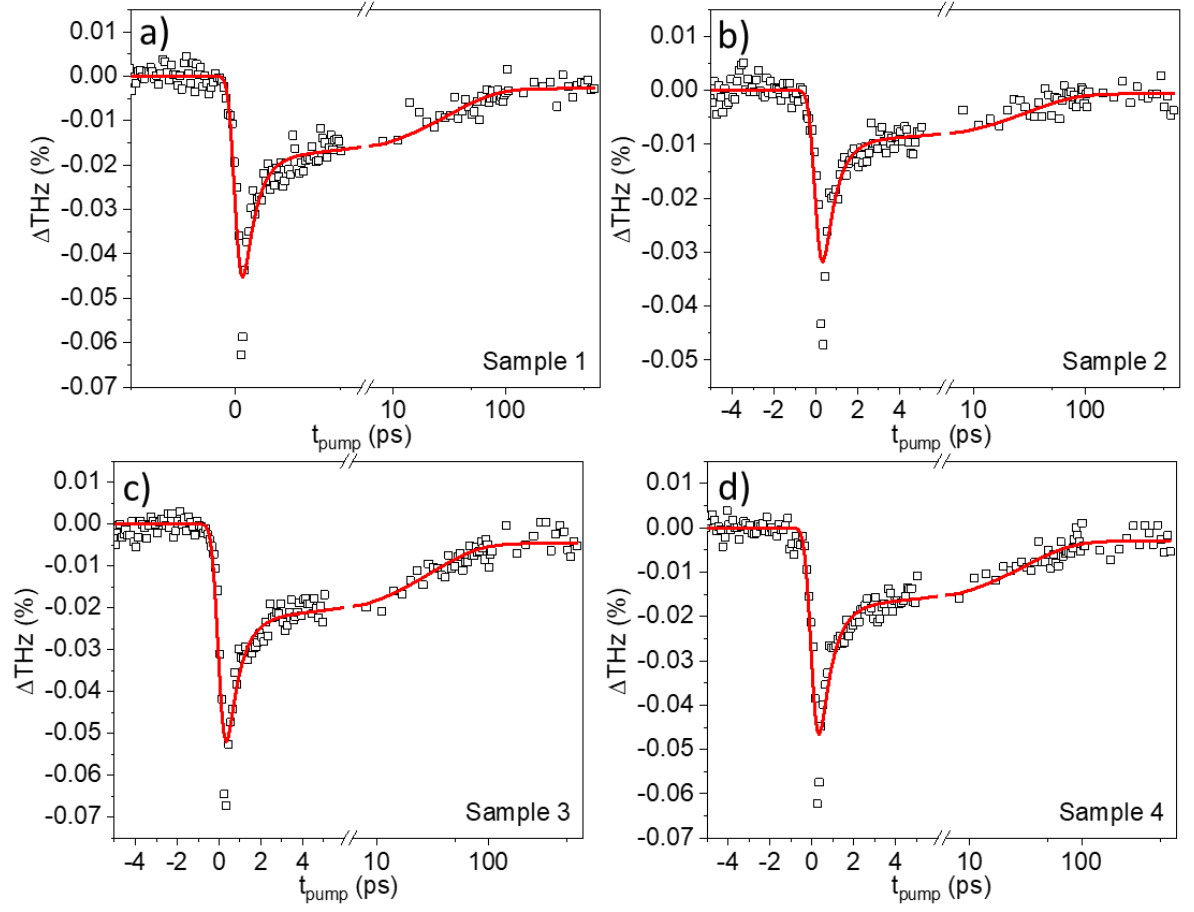

Figure S7. OPTP traces for four independent samples fit with a global Gaussian convoluted triexponential function. Parameters are shown in Table S1. 

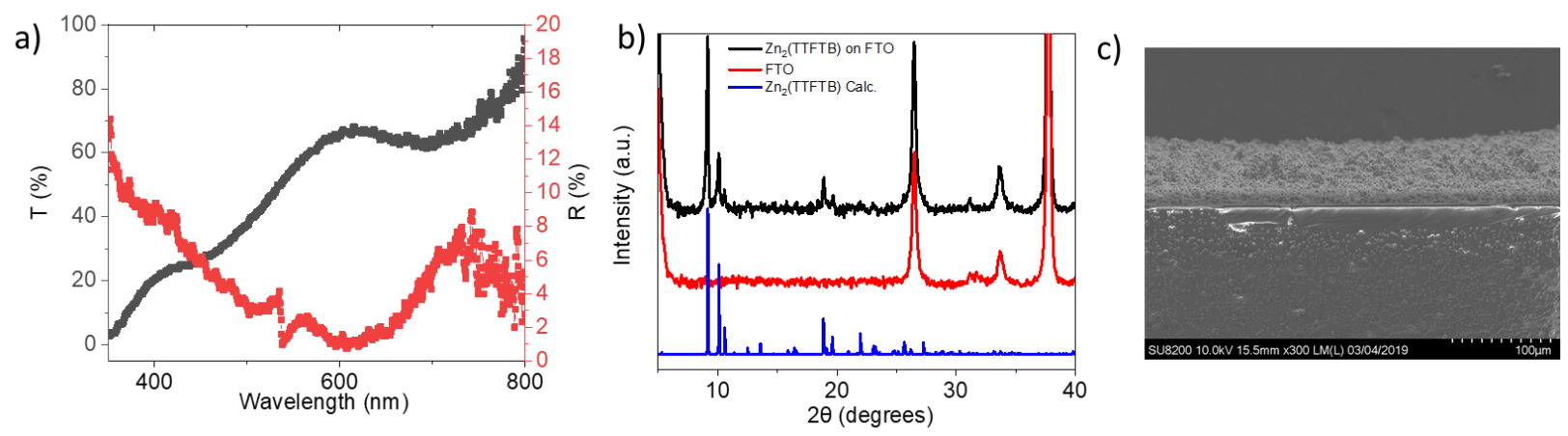

Figure S8. (a) UV-Visible transmission and diffuse reflection spectra. Penetration length of $6.9 \mu \mathrm{m}$ at $400 \mathrm{~nm}$ was calculated using the values of $8 \% \mathrm{R}$ and $20 \% \mathrm{~T}$. (b) pXRD pattern confirming correct crystallinity of $Z n_{2}$ TTFTB thin film on FTO. FTO-coated glass was required as a substrate for homogeneous attachment of $\mathrm{Zn}_{2}$ TTFTB film. (c) SEM side view image for transparent $\mathrm{Zn}_{2}$ TTFTB film grown on FTO showing $10 \mu \mathrm{m}$ thickness.
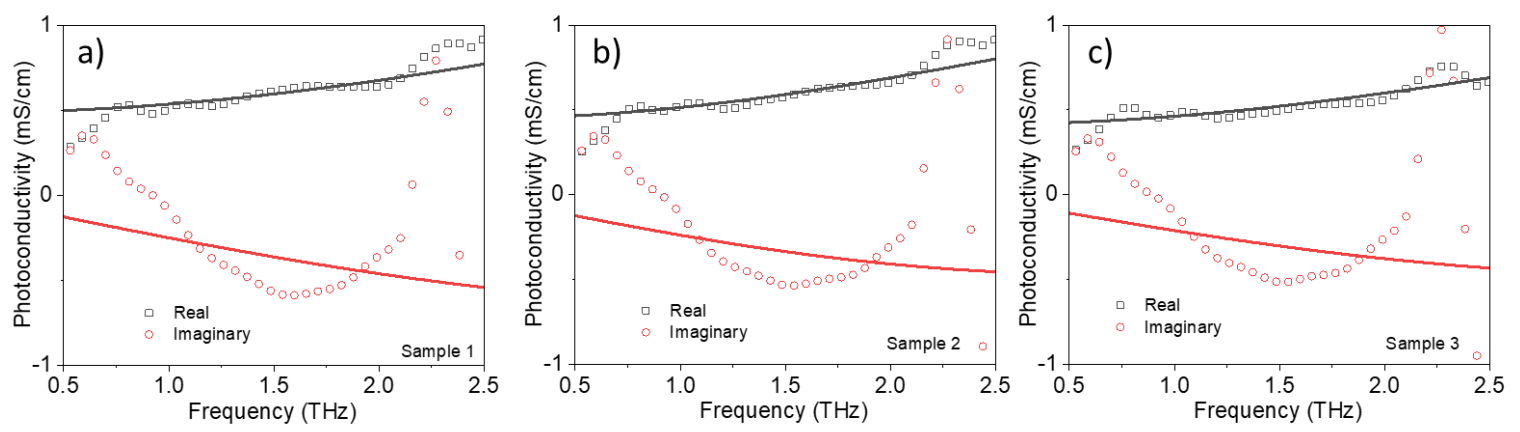

Figure S9. Drude-Smith fits for photoconductivity data of individual samples. 

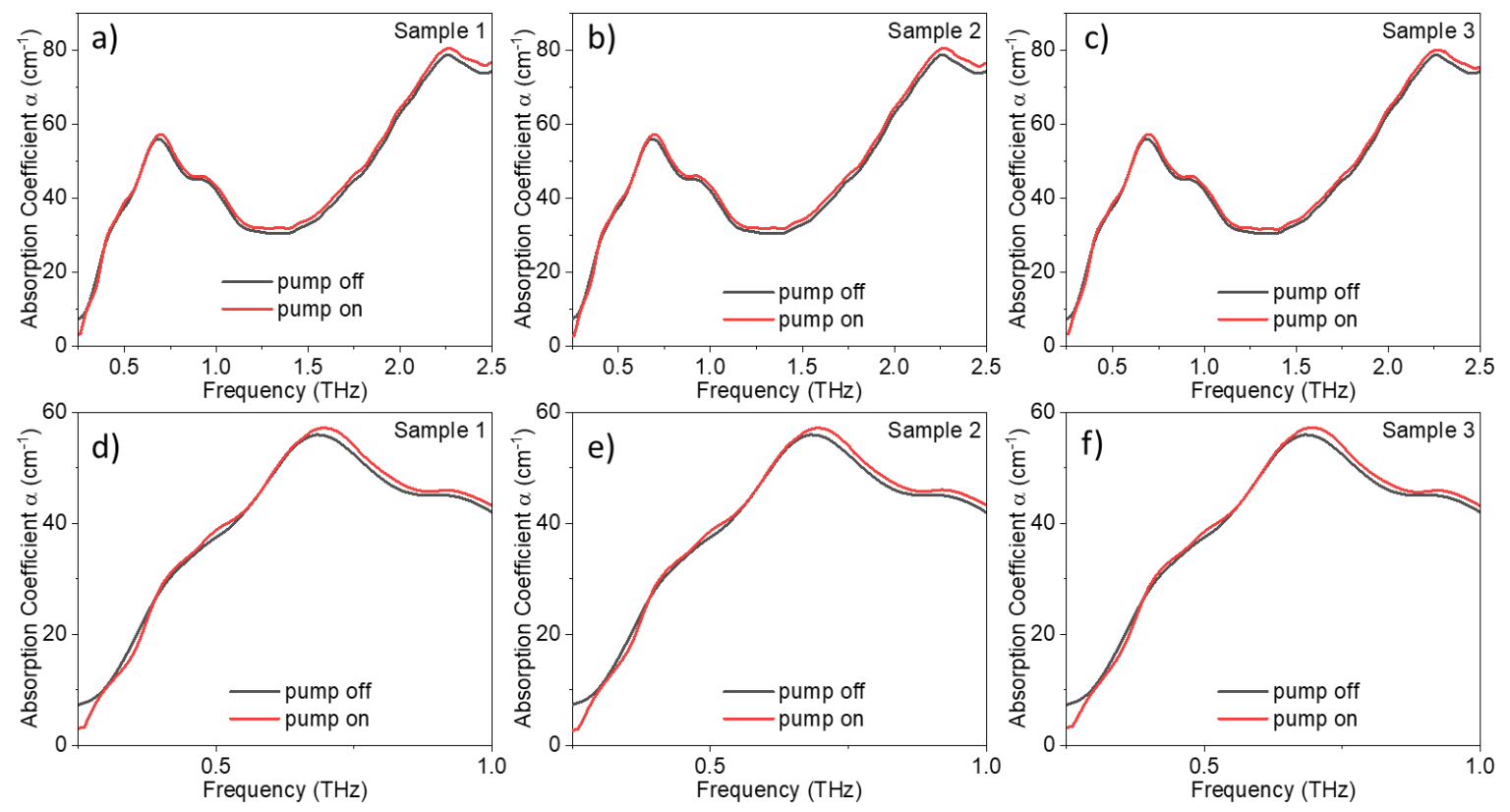

Figure S10. (a-c) The absorption coefficient with and without pump excitation in TRTS experiments for the three measured samples. At frequencies below $0.6 \mathrm{THz}$, photoexcitation results in resonance changes that complicate the extraction of photoconductivity. (d-e) show the expanded region from 0.3 to $1 \mathrm{THz}$ for each sample where photoinduced resonance changes are observed. Pump off data was determined according to equation 1 and pump on data was determined according to equation 5 .

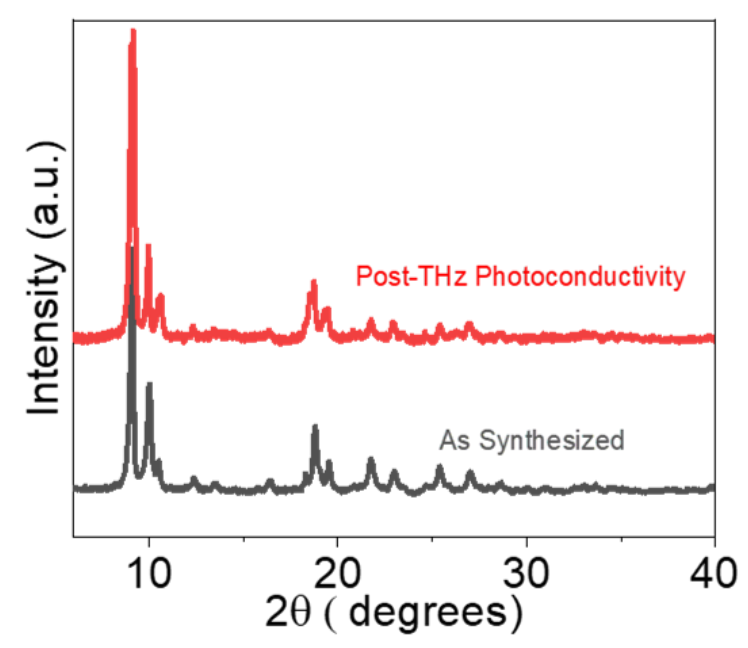

Figure S11. Post-TRTS pXRD confirming phase stability after beam exposure. 


\section{Supplementary Tables}

Table S1. OPTP fitting results for each individual sample. During fitting, time constants were fit globally, and their standard error is shown as the uncertainty. For each individual sample, the amplitudes were allowed to fit to account for slight differences in the overall trace amplitude. The $>>$ in $\tau_{3}$ is to clarify that the fixed time constant is much larger than the measurement time window.

\begin{tabular}{|c|c|c|c|c|c|c|}
\hline Sample & $\tau_{1}(p s)$ & $A_{1}(\%)$ & $\tau_{2}$ (ps) & $A_{2}(\%)$ & $\tau_{3}(p s)$ & $A_{3}(\%)$ \\
\hline 1 & \multirow{4}{*}{$0.60 \pm 0.03$} & 74.4 & \multirow{4}{*}{$31 \pm 3$} & 21.7 & \multirow{4}{*}{$\begin{array}{l}>>(10,000 ; \\
\quad \text { fixed })\end{array}$} & 3.9 \\
\hline 2 & & 82.2 & & 16.6 & & 1.2 \\
\hline 3 & & 72.1 & & 22.3 & & 5.6 \\
\hline 4 & & 76.4 & & 19.6 & & 4.0 \\
\hline
\end{tabular}

Table S2. Average Drude-Smith parameters with standard deviation uncertainties for the average of the three fit results.

\begin{tabular}{|cc|c|cc}
\hline Parameter & Sample 1 & Sample 2 & Sample 3 & Average \\
\hline $\mathrm{N}\left(10^{15} \mathrm{~cm}^{-3}\right)$ & 11.90 & 9.22 & 9.05 & $10 \pm 2$ \\
$\tau_{\text {scatt }}(\mathrm{fs})$ & 14.46 & 19.44 & 16.87 & $17 \pm 2$ \\
c parameter & -0.87 & -0.85 & -0.86 & $-0.86 \pm 0.01$ \\
\hline
\end{tabular}

\section{References}

(1) Mitamura, Y.; Yorimitsu, H.; Oshima, K.; Osuka, A. Chem. Sci. 2011, 2, 2017.

(2) Narayan, T. C.; Miyakai, T.; Seki, S.; Dincă, M. J. Am. Chem. Soc. 2012, 134, 12932.

(3) Neu, J.; Schmuttenmaer, C. A. J. Appl. Phys. 2018, 124, 231101.

(4) Neu, J.; Nikonow, H.; Schmuttenmaer, C. A. J. Phys. Chem. A 2018, 122, 5978.

(5) Neu, J.; Nemes, C. T.; Regan, K. P.; Williams, M. R. C.; Schmuttenmaer, C. A. Phys. Chem. Chem. Phys. 2018, 20, 276.

(6) Beard, M. C.; Turner, G. M.; Schmuttenmaer, C. A. Phys. Rev. B 2000, 62, 15764.

(7) Chu, C.; Huang, D.; Zhu, Q.; Stavitski, E.; Spies, J. A.; Pan, Z.; Mao, J.; Xin, H. L.; Schmuttenmaer, C. A.; Hu, S.; Kim, J.-H. ACS Catal. 2019, 9, 626.

(8) Jiang, J.; Spies, J. A.; Swierk, J. R.; Matula, A. J.; Regan, K. P.; Romano, N.; Brennan, B. J.; Crabtree, R. H.; Batista, V. S.; Schmuttenmaer, C. A.; Brudvig, G. W. J. Phys. Chem. C 2018, 122, 13529.

(9) Cook, D. J.; Hochstrasser, R. M. Opt. Lett. 2000, 25, 1210.

(10) Bartel, T.; Gaal, P.; Reimann, K.; Woerner, M.; Elsaesser, T. Opt. Lett. 2005, 30, 2805.

(11) Wu, Q.; Zhang, X. C. Applied Physics Letters 1995, 67, 3523.

(12) Neu, J.; Regan, K. P.; Swierk, J. R.; Schmuttenmaer, C. A. Appl. Phys. Lett. 2018, 113, 233901.

(13) Smith, N. V. Phys. Rev. B 2001, 64, 155106.

(14) Turner, G. M.; Beard, M. C.; Schmuttenmaer, C. A. J. Phys. Chem. B 2002, 106, 11716. 